\title{
Crim1 suppresses left ventricular hypertrophy
}

\author{
LONG YANG $^{1 *}$, JIONGHONG HE $^{1 *}$, GUILING XIA $^{1 *}$, JUN YANG $^{1}$, \\ QIAN TANG ${ }^{1}$, YONGYAO YANG ${ }^{1}$ and JIUSHENG DENG ${ }^{2}$ \\ ${ }^{1}$ Department of Cardiology, Guizhou Provincial People's Hospital, Guiyang, Guizhou 550002, P.R. China; \\ ${ }^{2}$ Department of Pathology and Laboratory Medicine, Emory University School of Medicine, Atlanta, GA 30322, USA
}

Received November 2, 2018; Accepted May 2, 2019

DOI: $10.3892 /$ br.2019.1214

\begin{abstract}
Left ventricular hypertrophy is a leading cause of heart failure and sudden death. Cysteine-rich transmembrane bone morphogenetic protein regulator 1 (Crim1) is expressed at a high level in the heart and has a regulatory role in heart development. The present study aimed to test the hypothesis that Crim1 can have an inhibitory function on ventricular hypertrophy. Rat primary ventricular myocytes were stretched to induce myocyte hypertrophy, and treated with telmisartan or infected with Crim1-expressing recombinant adenovirus (Ad-Crim1). Rat ventricular hypertrophy was induced by abdominal aortic coarctation (AAC), and treated either with telmisartan or myocardial injection of Ad-Crim1 or empty adenovirus vector. The results showed that the expression of Criml decreased in the hypertrophic ventricle. The inhibition of angiotensin receptor type 1 (AT1R) by telmisartan in vitro and in vivo significantly increased the expression of Crim 1 in the left ventricle. The overexpression of Crim 1 by infection with Ad-Crim1 significantly inhibited stretch-induced ventricular myocyte hypertrophy in vitro. The overexpression of Crim1 by gavage with AT1R inhibitor telmisartan or myocardial injection of Ad-Crim1 markedly suppressed AAC-induced left ventricular hypertrophy in vivo. These results suggest that Crim1 has a suppressive function on ventricular hypertrophy and provides a novel therapeutic target for the treatment of cardiac hypertrophy.
\end{abstract}

\section{Introduction}

Left ventricular hypertrophy is characterized by abnormal thickness of the ventricular wall in response to increased heart

Correspondence to: Professor Long Yang, Department of Cardiology, Guizhou Provincial People's Hospital, 83 Zhongshandong Road, Guiyang, Guizhou 550002, P.R. China

E-mail: yanglong1001@outlook.com

*Contributed equally

Key words: left ventricular hypertrophy, abdominal aortic coarctation, cysteine-rich transmembrane bone morphogenetic protein regulator 1 , telmisartan workload, leading to heart failure and sudden death (1). The most common cause of ventricular hypertrophy is high blood pressure (2). Angiotensin II (AngII) is a key signaling molecule that induces ventricular myocyte hypertrophy through the activation of angiotensin receptor type 1 (AT1R) (3). Unlike the AngII-dependent pathway, stretch induces the translocation of $\beta$-arrestin 2 in ventricular myocytes. $\beta$-arrestin 2 binds and activates AT1R, inducing hypertrophic gene expression and the development of ventricular hypertrophy (4).

Cysteine-rich transmembrane bone morphogenetic protein regulator 1 (Crim1) is a type-I transmembrane protein, and is expressed in multiple types of cell and tissue, including the proepicardium, epicardium, central nervous system, vascular system and the heart (5-10). Crim1 serves an important role in vascular tube formation (6) and has cell-autonomous and paracrine roles during heart development (10). Deficiency of the CRIM1 gene causes perinatal mortality with defects in multiple organs, including hemorrhagic necrosis, enlargement of glomerular capillary lumens, congenital heart defects, epicardial dysplasia and ventricular myocardium densification dysplasia $(11,12)$. However, whether Crim1 is involved in left ventricular hypertrophy and whether the AT1R signaling pathway affects the expression of Crim1 in ventricular myocytes remain unclear. The present study investigated the suppressive function of Crim1 on left ventricular hypertrophy and the effect of AT1R signaling on the expression of Crim1 in ventricular myocytes.

\section{Materials and methods}

Isolation of primary ventricular myocytes. Primary ventricular myocytes were isolated from the left ventricles of neonatal Sprague-Dawley rats (4 male and 6 female, 1 day after birth) (purchased from Peking University Medical Center Animal Center, Beijing, China; approval no. SYXK2011-0039) as described previously (13). The rats were housed at room temperature $\left(20-25^{\circ} \mathrm{C}\right)$ with $40-60 \%$ relative humidity and a day-night cycle of $12 \mathrm{~h}$. Food and water were provided ad libitum. The rat ventricular muscle was digested with $0.01 \%$ trypsin and $0.03 \%$ type II collagenase at $37^{\circ} \mathrm{C}$ for 6 min (Sigma-Aldrich; Merck KGaA, Darmstadt, Germany), followed by differential adhesion and 5'-bromodeoxyuridine treatment. The attached cells were then cultured at $37^{\circ} \mathrm{C}$ for $48 \mathrm{~h}$ in DMEM (cat no. C11885500BT, Gibco; Thermo Fisher Scientific, Inc., Waltham, MA, USA) containing 10\% 
fetal bovine serum (FBS; cat no. 10099141, Gibco; Thermo Fisher Scientific, Inc.). Ventricular myocytes were identified by immunohistochemical staining with rabbit anti-rat $\alpha$-striated muscle actin ( $\alpha$-SCA) antibodies (1:100; cat. no. bs-0189R, Shanghai Kemin Bio-tech, Shanghai, China) for $1 \mathrm{~h}$ at room temperature, followed by incubation with horseradish peroxidase-conjugated goat anti-rabbit secondary antibodies (1:3,000; cat. no. SSA-004, Beijing Zhongyu Jinqiao Company, Beijing, China) for $1 \mathrm{~h}$ at room temperature. For immunofluorescent microscopy, the myocytes were fixed with $3.7 \%$ paraformaldehyde in phosphate-buffered saline (PBS) for $10 \mathrm{~min}$ at room temperature, and treated with $0.5 \%$ Triton X-100 (Sigma-Aldrich; Merck KGaA) in PBS for 15 min at room temperature. Following a wash with PBS, the cells were incubated with FITC-conjugated $\alpha$-SCA antibodies (Shanghai Kemin Bio-tech, China) for $30 \mathrm{~min}$ at room temperature. Following further washing with PBS, the cells were examined using an LSM 410 confocal microscope (Zeiss AG, Oberkochen, Germany).

Cell stretch and transfection in vitro. Following culture in DMEM containing $10 \% \mathrm{FBS}$ at $37^{\circ} \mathrm{C}$ for $48 \mathrm{~h}$, the primary left ventricular myocytes were cultured in serum-free DMEM and treated with $10 \mu \mathrm{M}$ telmisartan (Medicinal product name: Micardis; AT1R inhibitor; Sigma-Aldrich; Merck KGaA) at $37^{\circ} \mathrm{C}$ for $2 \mathrm{~h}$, followed by $24 \mathrm{~h}$ of cell stretch. Cell stretch was performed in silicone dishes (Strex Cell; $10^{5}$ cells/dish) coated with collagen $(150 \mu \mathrm{g} / \mathrm{ml})$. The cells were exposed to $20 \%$ cyclic stretch in uniaxial strain at 30 cycles/min using a computer-controlled stepping motor (Strex Cell). Cells without exposure to stretch or telmisartan treatment served as a control. Alternatively, primary ventricular myocytes $\left(10^{4}\right.$ cells/well $)$ were seeded in wells of 12-well tissue culture plates and transfected with recombinant adenovirus expressing Crim1 (Ad-Crim1; Thermo Fisher Scientific, Inc.) in DMEM with $10 \%$ FBS at $37^{\circ} \mathrm{C}$ for $36 \mathrm{~h}$ using Lipofectamine ${ }^{\circledR} 2000$ (cat. no. 18324-111, Invitrogen; Thermo Fisher Scientific, Inc.) according to the manufacturer's protocols. The multiplicity of infection (MOI) of Ad-Crim1 was 25, 100 or 200 (active viral particles per myocyte). Empty virus (Ad-null) at MOI=100 served as a control. The transfected cells were then harvested, and washed with centrifugation at $178 \mathrm{x}$ g for $5 \mathrm{~min}$ at room temperature. The cell pellet was suspended in PBS with $0.25 \%$ SDS, and aliquoted for protein or DNA measurement. Protein was quantified using the bicinchoninic acid method (Sigma-Aldrich; Merck KGaA). DNA was measured using a Quant-iTTM dsDNA assay kit (Invitrogen; Thermo Fisher Scientific, Inc.). The protein/DNA ratio was then calculated as an indicator of myocyte hypertrophy.

Histochemistry. The left ventricular myocytes were stained with crystal violet, as described previously (14). Changes in cell size were analyzed with ImageJ software (Version 1.4, National Institutes of Health) using a Leica DM300 binocular microscope (Leica Microsystems GmbH, Wetzlar, Germany). Alternatively, paraffin-embedded myocardial sections $(5 \mu \mathrm{m})$ were subjected to hematoxylin and eosin (H\&E) staining, and images were captured under a microscope. The cross-sectional area of the ventricular myocytes was analyzed using ImageJ software. A total of 50 ventricular myocytes per group were analyzed and the mean value was calculated.

Reverse transcription-quantitative polymerase chain reaction $(R T-q P C R)$ analysis. RNA was isolated from the rat left ventricle or ventricular myocytes using TRIzol reagent, as described previously (15). The mRNA expression of Crim1 was measured by RT-qPCR analysis (15). Briefly, total RNA $(1.5 \mu \mathrm{g})$ was subjected to reverse transcription with TransScript ${ }^{\circledR}$ First-Strand cDNA Synthesis SuperMix. The reverse transcribed products $(2 \mu \mathrm{g})$ were used for qPCR with $\operatorname{TransStart}^{\circledR}$ PT Green qPCR SuperMix and primers (Beijing Golden Biotechnology Co., Ltd., Beijing, China; Table I). The PCR program was as follows: $95^{\circ} \mathrm{C}$ pre-denaturation for $10 \mathrm{~min}$, followed by 40 cycles of $95^{\circ} \mathrm{C}$ denaturation for $30 \mathrm{sec}, 60^{\circ} \mathrm{C}$ annealing for $30 \mathrm{sec}$, and $72^{\circ} \mathrm{C}$ extension for $30 \mathrm{sec}$. The glyceraldehyde-3-phosphate dehydrogenase (GAPDH) gene was used as an internal control. Relative gene expression was calculated using the $2^{-\Delta \Delta C q}$ method (16).

Western blot analysis. Total protein was extracted from the left ventricle or ventricular myocytes with RIPA lysis buffer containing protease inhibitors, as described previously (17). Protein concentration was quantified using the bicinchoninic acid method. The proteins isolated from myocytes $(40 \mu \mathrm{g})$ or the left ventricle $(100 \mu \mathrm{g})$ were separated on 6\% SDS-PAGE and transferred onto PVDF membranes (Bio-Rad Laboratories, Inc. Hercules, CA, USA). The membranes were incubated with rabbit anti-rat Crim1 (1:100; cat. no. bs-2034R, Beijing Boao Sen Company, Beijing, China) or anti-GAPDH (1:200; cat. no. sc-365062, Santa Cruz Biotechnology, Inc., Dallas, TX, USA) primary antibodies for $1 \mathrm{~h}$ at room temperature, followed by incubation with horseradish peroxidase-conjugated goat anti-rabbit secondary antibodies (1:3,000; cat. no. SSA-004, Beijing Zhongyu Jinqiao Company) for $1 \mathrm{~h}$ at room temperature. The protein bands were detected with a Bio-Rad chemiluminescence detector and analyzed with ImageJ software.

Animal experiments. Male Sprague-Dawley rats (8 weeks, weight 220-250 g) were purchased from the Peking University Medical Center Animal Center (Beijing, China), and housed at room temperature $\left(20-25^{\circ} \mathrm{C}\right)$ with $40-60 \%$ relative humidity under a $12 \mathrm{hlight} / \mathrm{dark}$ cycle. Food and water were provided ad libitum. Rats were weighed and subjected to sham operation with saline or telmisartan $(3.57 \mathrm{mg} / \mathrm{kg} / \mathrm{d}$; Sigma-Aldrich; Merck KGaA) gavage, abdominal aortic coarctation (AAC) (18) with saline gavage, or AAC with telmisartan gavage $(3.57 \mathrm{mg} / \mathrm{kg} / \mathrm{d})$. The duration of abdominal aortic coarctation was 1 week, and treatment with telmisartan lasted for 8 weeks. Alternatively, the rats received a sham operation with saline intramuscular injection, $\mathrm{AAC}+$ saline intramuscular injection, $\mathrm{AAC}+\mathrm{Ad}-$ null or Ad-Crim1 intramuscular injection. Each group included 10 rats. All rats were provided by the Peking University Medical Center Animal Center (Beijing, China). The use of rats for experiments was approved by the Ethics Review Committee of Guizhou Provincial People's Hospital (Guizhou, China).

$A A C$. The rats were fasted for $8 \mathrm{~h}$ and subjected to abdominal anesthesia with $10 \%$ chloral hydrate $(300 \mathrm{mg} / \mathrm{kg}$ of body weight) and laparotomy. Ligation was performed on the 
Table I. Reverse transcription-quantitative polymerase chain reaction primers.

\begin{tabular}{lll}
\hline Gene & \multicolumn{1}{c}{ Forward (5'-3') } & Reverse (5'-3') \\
\hline Crim1 & GTCTTTCCCCGGCGATCA & TTGTTGCAGGTTCGGATGGT \\
GAPDH & GTCAGTGGTGGACCTGGACCT & AGGGGAGATTCAGTGTGGTG
\end{tabular}

Crim1, cysteine-rich transmembrane bone morphogenetic protein regulator 1; GAPDH, glyceraldehyde-3-phosphate dehydrogenase.

abdominal aorta above the renal artery bifurcation $(\sim 1 \mathrm{~cm}$ long) by placing a blunt needle (22-G). The duration of AAC was 7 days, and the abdominal aorta was partially narrowed to $\sim 0.7 \mathrm{~mm}$ diameter. Animals in the sham operation group underwent laparotomy only, without abdominal aorta ligation.

Intramuscular injection of adenovirus. The rats were anesthetized 1 week following abdominal surgery, and were subjected to intramuscular injection of Ad-null or Ad-Crim1 (3x10 ${ }^{9} \mathrm{PFU}$ in $200 \mu \mathrm{l}$ saline) into the left ventricle. The rats were subjected to echocardiography 8 weeks following viral injection.

Echocardiography. The rats were anesthetized with 1.0-1.5\% isoflurane 9 weeks following abdominal surgery, and subjected to M-mode echocardiography using a high-resolution echocardiographic system (Sequoia 512; Acuson, Siemens, Munich, Germany). The thickness of the left ventricular wall and the diameter of the left ventricle were measured using the Vevo2100 ultrasound system. The weight of the left ventricle was calculated using the following formula: (IVSd + LVDd + PWDd $)^{3}-\mathrm{LVDd}^{3}$, in which IVSd is the ventricular diastolic thickness, LVDd is the left ventricular diastolic diameter, and PWDd is the left ventricular posterior wall diastolic thickness.

Left ventricle harvesting. The heart was immediately harvested following cardiac ultrasound. The left ventricle was weighed following removal of the atrium and the right ventricle (remaining room interval). The left ventricle was fixed with $4 \%$ paraformaldehyde for paraffin embedding, or frozen for the extraction of proteins or RNA.

Statistical analysis. All data are presented as the mean \pm standard deviation, and were analyzed by Student's t-test or Tukey's honestly significant difference post hoc test following one-way ANOVA with GraphPad Prism 6 software (GraphPad Software, Inc.). Each experiment was performed with three repetitions. $\mathrm{P}<0.05$ was considered to indicate a statistically significant difference.

\section{Results}

Expression of Criml is decreased in hypertrophic ventricular myocytes. Immunohistochemical staining and confocal microscopy demonstrated that primary ventricular myocytes were $\alpha$-SCA-positive (Fig. 1A). Quantification of protein and DNA in ventricular myocytes treated with or without stretch (19) revealed that hypertrophic myocytes induced by stretch had a significantly higher protein/DNA ratio than those without stretch (Fig. 1B). Analysis of the mRNA expression of
Crim1 by RT-qPCR analysis (Fig. 1C) and protein expression by western blot analysis (Fig. 1D) demonstrated that the mRNA and protein expression levels of Crim1 were significantly lower in the hypertrophic ventricular myocytes compared with those in the unstretched cells (Fig. 1C and D).

Increased expression of Criml abrogates left ventricular myocyte hypertrophy in vitro. Activating AT1R signaling promotes left ventricular hypertrophy (20). To determine whether inhibiting AT1R signaling can have an effect on the expression of Crim 1 in ventricular myocytes, the stretched primary ventricular myocytes were treated with AT1R antagonist telmisartan. The RT-qPCR assay demonstrated that telmisartan treatment significantly increased the mRNA expression of Crim1 in the stretched myocytes compared with that in the saline treatment group (Fig. 2A). Western blot analysis further demonstrated that telmisartan treatment significantly enhanced the protein expression of Crim1 in cells (Fig. 2B). Consequently, telmisartan treatment abrogated stretch-induced ventricular myocyte hypertrophy, with a significant decrease in the protein/DNA ratio in the cells. The average protein/DNA ratio in the control cells and telmisartan-treated cells were 2.5 and 1.7 respectively (Fig. 2C). To determine whether the overexpression of Crim1 had an inhibitory effect on ventricular myocyte hypertrophy, primary left ventricular myocytes were transfected with Ad-Crim1 or Ad-null. Subsequent western blot analysis revealed that transfection with Ad-Crim1 enhanced the expression of Crim1 in the transfected ventricular myocytes, compared with that in the Ad-null treatment group (Fig. 3A). To examine the suppressive function of Crim1 on stretch-induced myocyte hypertrophy, crystal violet staining was performed on the stretched myocytes. Histochemical analysis demonstrated that Ad-Crim1 transfection (MOI=100) markedly reduced myocyte hypertrophy, compared with Ad-null treatment or no viral transfection. Ad-null transfection had no suppressive effect on myocyte hypertrophy (Fig. 3B). Analysis of the protein/DNA ratio demonstrated that Ad-Crim1 treatment significantly reduced the ratio in the stretched myocytes, compared with Ad-null treatment or no viral transfection (Fig. 3C). The average protein/DNA ratios in the stretched cells treated with Ad-Crim1, Ad-null, and without viral transfection were 1.3, 1.9 and 2.1 respectively (Fig. 3C). The average protein/DNA ratio in the control myocytes without stretch was 1.15 (Fig. 3C). There was no significant difference in the protein/DNA ratio between the stretch/Ad-Crim1-treated myocytes and the control cells (Fig. 3C). Measurements of cell size with Image $\mathbf{J}$ software further confirmed that the overexpression of Crim1 by transfection with Ad-Crim1 significantly reduced 
A

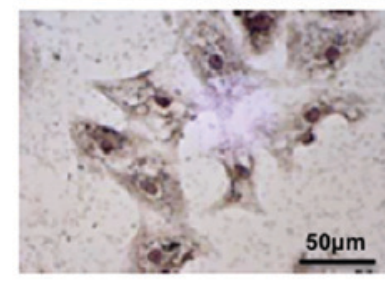

B

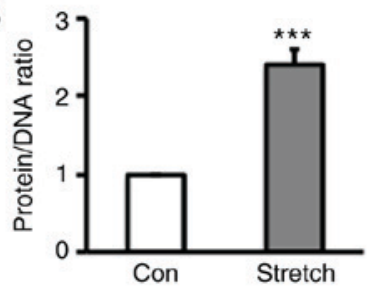

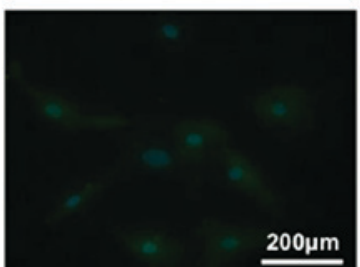

C

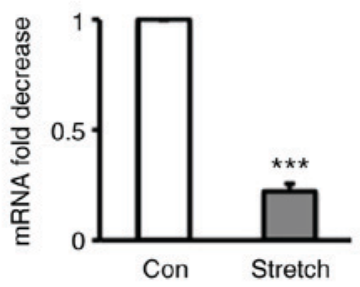

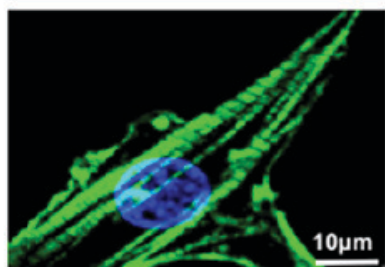

D

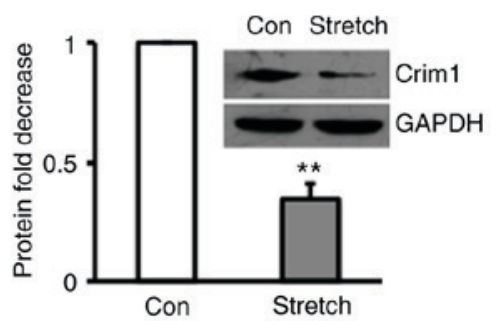

Figure 1. Expression of Crim1 in rat hypertrophic ventricular myocytes. (A) Primary myocytes isolated from left ventricle were identified by immunohistochemical staining with $\alpha$-SCA antibodies (left) or immunofluorescent staining with FITC-conjugated $\alpha$-striated muscle actin antibodies (middle and right), with images captured under a confocal microscope (magnifications, $\mathrm{x} 4$ and 200). Primary left ventricular myocytes were exposed to stretch, with cells without stretch serving as a control. (B) Protein/DNA ratio in stretched or control cells was profiled. (C) mRNA and (D) protein expression levels of Crim1 were examined by reverse transcription-quantitative polymerase chain reaction analysis and western blotting, respectively. GAPDH served as internal control. Expression of Crim1 was calculated as the fold decrease compared with the control. Data were from three independent experiment, presented as the mean $\pm \mathrm{SD}$, and analyzed using Student's t-test. ${ }^{* * *} \mathrm{P}<0.01,{ }^{* * * *} \mathrm{P}<0.001$ vs. Con. Con, control. Crim1, cysteine-rich transmembrane bone morphogenetic protein regulator 1; GAPDH, glyceraldehyde-3-phosphate dehydrogenase; Con, control.

A

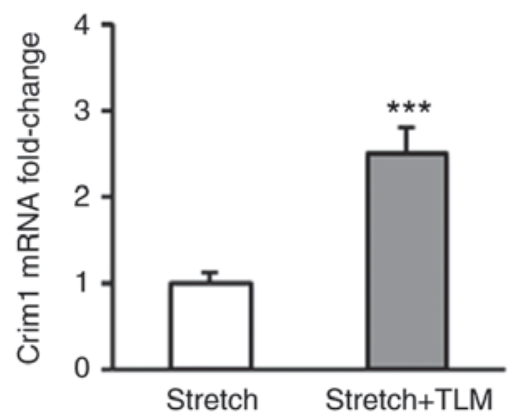

B

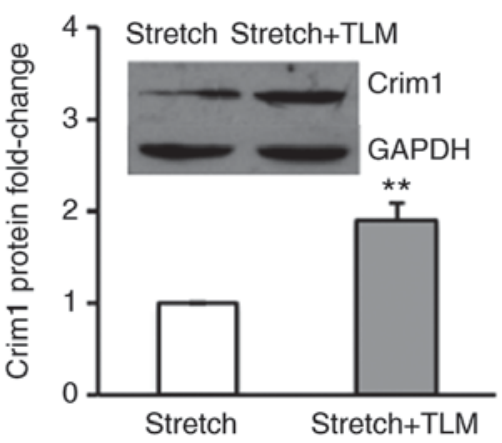

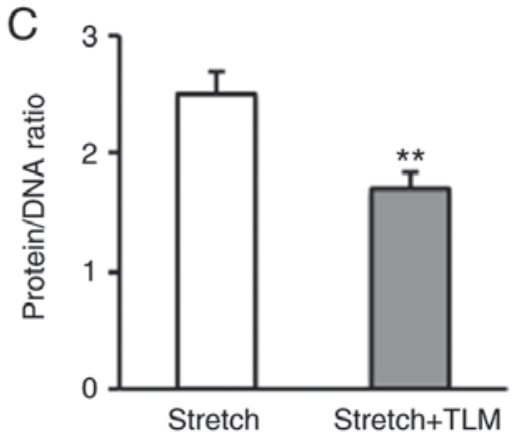

Figure 2. Effect of telmisartan treatment on the expression of Crim1 in rat hypertrophic ventricular myocytes. Stretched primary left ventricular myocytes were treated with TLM. (A) mRNA and (B) protein expression levels of Crim1 were examined by reverse transcription-quantitative polymerase chain reaction analysis and western blotting, respectively. GAPDH served as an internal control. (C) Protein/DNA ratio in the cells. Expression levels of Crim1 in TLM-treated ventricular myocytes was calculated as the fold increase comparison with those without TLM. Data were from three independent experiments, presented as the mean $\pm \mathrm{SD}$, and analyzed using Student's t-test. ${ }^{* * *} \mathrm{P}<0.01,{ }^{* * * *} \mathrm{P}<0.001$ vs. Stretch. Crim1, cysteine-rich transmembrane bone morphogenetic protein regulator 1; GAPDH, glyceraldehyde-3-phosphate dehydrogenase; TLM, telmisartan.

the cell size and inhibited ventricular myocyte hypertrophy, compared with Ad-null treatment and no viral transfection (Fig. 3D).

Enhancement of Crim1 by telmisartan inhibits left ventricular myocyte hypertrophy in vivo. To examine whether enhancement of the protein expression of Crim1 can suppress AAC-induced left ventricular myocyte hypertrophy, the AAC-treated rats were subjected to telmisartan gavage and the protein expression of Crim1 in the left ventricle was analyzed. Western blot analysis demonstrated that the protein expression of Crim1 was significantly decreased in the left ventricle of the AAC-treated rats, compared with that in the sham group (Fig. 4A). However, protein expression of Crim1 in the left ventricle of the AAC-treated rats with telmisartan gavage was significantly increased compared with that in the group without telmisartan treatment (Fig. 4A). H\&E staining further demonstrated that telmisartan treatment abrogated AAC-induced left ventricular myocyte hypertrophy compared with that in the absence of telmisartan treatment (Fig. 4B). Quantification of myocyte cross-sectional area further confirmed that the size of ventricular myocytes in AAC-treated rats with telmisartan gavage was significantly reduced compared with that in the rats without telmisartan treatment (Fig. 4C). The expression of Crim1 and myocyte size in the left ventricle of AAC/telmisartan-treated rats did not differ significantly from those in the sham group or in the rats without AAC but with telmisartan treatment (Fig. 4A-C).

Injection of Ad-Criml suppresses AAC-induced left ventricular hypertrophy in vivo. To examine whether the administration of Ad-Crim1 suppresses AAC-induced left 
A
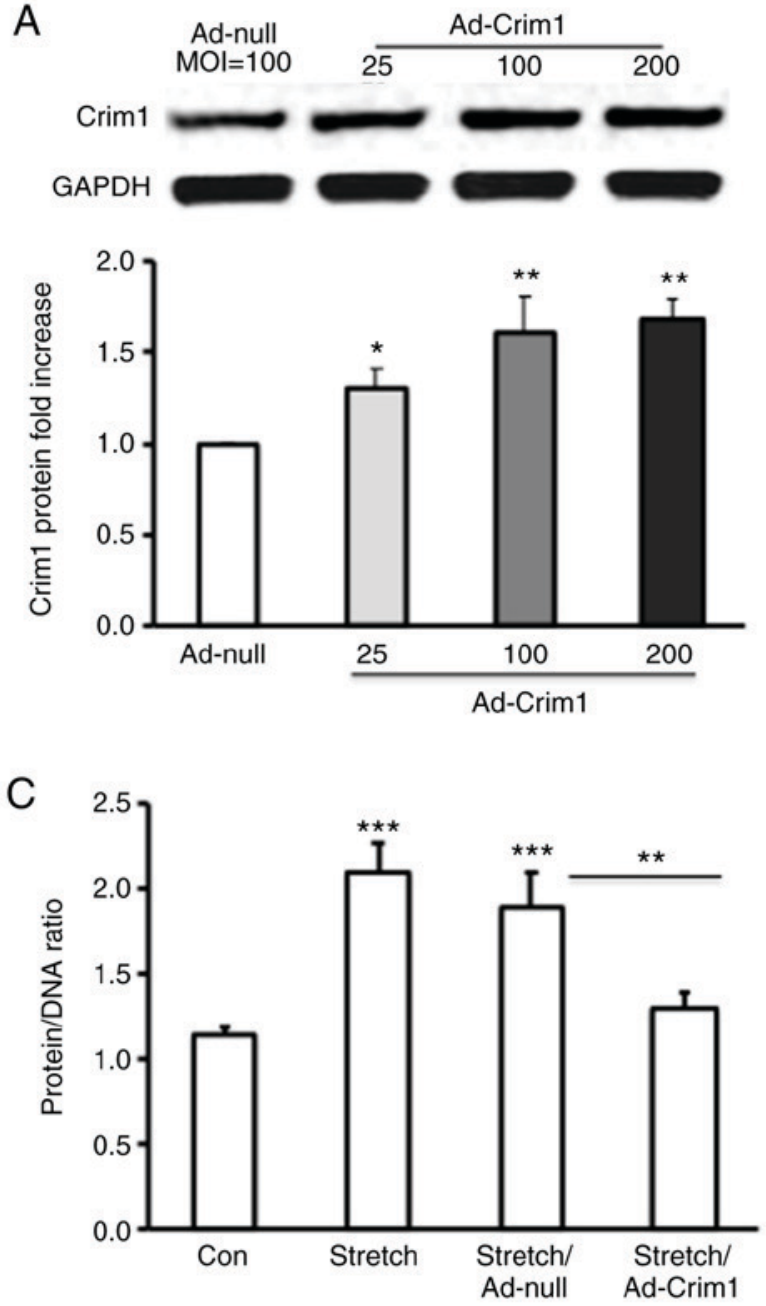

B
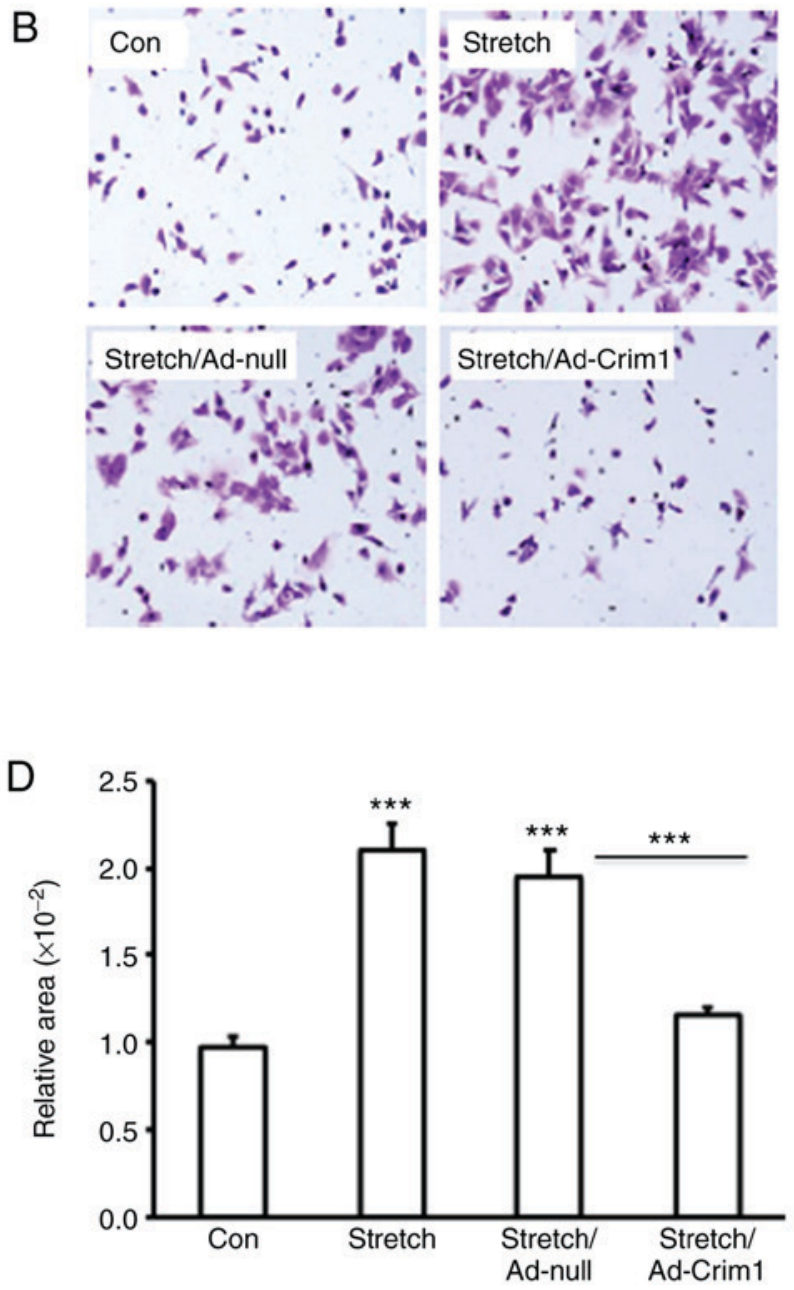

Figure 3. Effect of the overexpression of Crim1 on left ventricular myocyte hypertrophy. (A) Primary rat left ventricular myocytes were infected with Ad-Crim1 or Ad-null. Proteins were isolated from the treated cells and subjected to western blotting with anti-Crim1 or GAPDH specific antibodies. Protein expression levels of Crim1 in Ad-Crim1-treated cells are presented as the fold increase compared with Ad-null-treated cells. (B) Primary left ventricular myocytes were subjected to stretch and transfected with Ad-Crim1, Ad-null, or without transfection. Ventricular myocytes without treatment served as a control. The cells were then stained with crystal violet and images were captured under a microscope (magnification, x20). (C) Protein/DNA ratio in treated or control ventricular myocytes. (D) Cross-sectional area of ventricular myocytes was analyzed using ImageJ software. Data are from three independent experiments, presented as the mean $\pm \mathrm{SD}$ and analyzed by one-way ANOVA. ${ }^{*} \mathrm{P}<0.05,{ }^{* *} \mathrm{P}<0.01,{ }^{* * *} \mathrm{P}<0.001$. Individual treatment was compared with the control; stretch/Ad-Crim1 treatment was also compared with stretch/Ad-null treatment. Crim1, cysteine-rich transmembrane bone morphogenetic protein regulator 1; GAPDH, glyceraldehyde-3-phosphate dehydrogenase; MOI, multiplicity of infection; Con, control.

ventricular hypertrophy in the rat model, as Ad-Crim1 did on primary ventricular myocytes in vitro (Fig. 3B-D), the AAC-treated rats were subjected to intramuscular injection of Ad-Crim1, Ad-null or saline. Echocardiography (Fig. 5A) demonstrated that the left ventricular end-diastolic anterior wall (LAVWd) in the AAC-treated rats was significantly thicker compared with that in the sham group (Fig. 5B). There were no significant differences in LAVWd among the three groups of rats treated with Ad-Crim1, Ad-null or without viral injection (Fig. 5B). Measurement of the left ventricular mass revealed that AAC treatment significantly increased left ventricular weight compared with that following sham treatment (Fig. 5C). However, rats treated with Ad-Crim1 had significantly lower left ventricular weight compared with the those treated with Ad-null or without viral injection (Fig. 5C). Quantification of myocyte cross-sectional area further confirmed that AAC treatment markedly increased the size of ventricular myocytes compared with that in the sham treatment group (Fig. 5D); Ad-Crim1 treatment significantly reduced the size of left ventricular myocytes compared with that in the Ad-null treatment or without viral injection groups (Fig. 5D). There was no significant difference in myocyte size between rats treated with Ad-null and without viral injection (Fig. 5D). H\&E staining of the left ventricle confirmed that AAC treatment induced ventricular myocyte hypertrophy compared with that in the sham treatment group (Fig. 5E). Intramuscular injection of Ad-Crim1 markedly suppressed AAC-induced myocyte hypertrophy compared with that in the Ad-null treatment group (Fig. 5E).

\section{Discussion}

In the present study, it was demonstrated that Crim1 had an inhibitory effect on left ventricular hypertrophy. Enhancement of the expression of Crim 1 in myocytes abrogated left ventricular hypertrophy. 
A
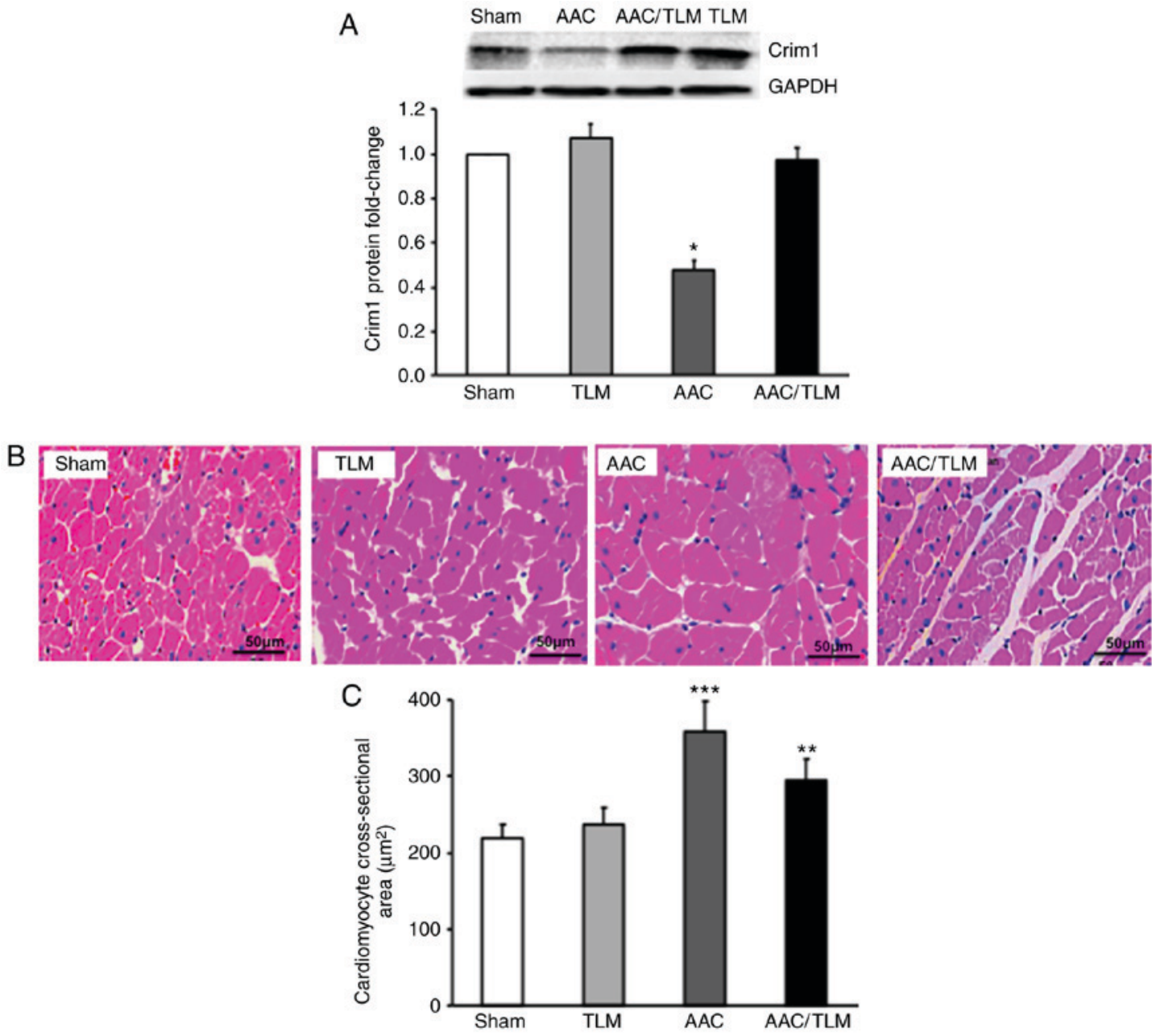

Figure 4. Effects of TLM on the expression of Crim1 and ventricular hypertrophy in vivo. Rats were subjected to AAC treatment with (AAC/TLM) or without (ACC) TLM gavage, or TLM gavage without AAC (TLM), or sham treatment. (A) Proteins were extracted from left ventricle of the four groups of rats and subjected to western blotting with anti-Crim1 or anti-GAPDH antibodies. GAPDH served as the internal protein control. The fold decrease or increase in Crim 1 protein in the TLM-, AAC-or AAC/TLM-treated groups were compared with the sham group. (B) Paraffin-fixed left ventricular sections from the four groups of rats were subjected to hematoxylin and eosin staining and images were captured under a confocal microscope (magnification, $\mathrm{x} 400, \mathrm{scale}$ bar $=50 \mu \mathrm{m}$ ). (C) Cross-sectional area of left ventricular myocytes was analyzed using ImageJ software. Data were from three independent experiments, presented as the mean $\pm \mathrm{SD}$, and analyzed using one-way ANOVA. ${ }^{*} \mathrm{P}<0.05,{ }^{* *} \mathrm{P}<0.01,{ }^{* * *} \mathrm{P}<0.001$. Individual treatment was compared with sham; AAC/TLM treatment was also compared with AAC treatment. Crim1, cysteine-rich transmembrane bone morphogenetic protein regulator 1; GAPDH, glyceraldehyde-3-phosphate dehydrogenase; TLM, telmisartan; AAC, abdominal aortic coarctation.

Crim1 protein has six conserved domains of chordin-like von Willebrand C-type cysteine-rich repeats (CRR) (5), existing in the Golgi, endoplasmic reticulum and on the cell surface (6,7). Crim1 CRR domains can intracellularly bind several cystine knot-containing growth factors, including vascular endothelial growth factor $\alpha(\mathrm{VEGF} \alpha)$, bone morphogenetic protein (BMP)2 and BMP4, and these molecules are antagonized through reducing its expression, processing and secretion $(7,21,22)$. The activation of BMP2 or BMP4 promotes the development of cardiac hypertrophy (23-25). Triggering VEGF $\alpha$ signal transduction facilitates tissue growth and angiogenesis in cardiac hypertrophy $(26,27)$. It is known that Crim1 inhibits BMP signaling by either limiting BMP precursors on the cell surface or preventing the maturation or secretion of BMP (7). Data from the present study demonstrated that the increased expression of Crim1 in cardiomyocytes by infection with Ad-Crim1 suppressed left ventricular hypertrophy. One of the mechanistic actions of Crim1 in suppressing ventricular/cardiac hypertrophy may be through inhibiting the activation of BMP2 or BMP4 signaling. Crim1 can also maintain retinal vascular and renal microvascular stability by regulating VEGF $\alpha$ signal transduction in vascular endothelial cells $(22,28)$. It is possible that the inhibitory effect of Crim1 on ventricular hypertrophy is mediated by affecting the delivery and function of VEGF $\alpha(21,22)$. Crim1 can also bind to $\beta$-integrin and regulate its signaling (29). Cardiomyocytes can undergo biomechanical change through receptors, including integrins (30). $\beta$-integrin, a mechanical sensor, can transduce mechanical force into biological information in collapsible muscle cells (31). $\beta$-integrin is also important in heart self-protection and the compensatory reaction in response to stress. The data obtained in the present study demonstrated that Crim1 prevented stretch-induced left ventricular hypertrophy. It is possible that the inhibitory function of Crim1 on myocardial hypertrophy may be through regulating biological stress-induced $\beta$-integrin signal transduction.

AT1R is a major pathogenic molecule contributing to cardiac damage (32). The overstimulation of AT1R causes 


\section{A}
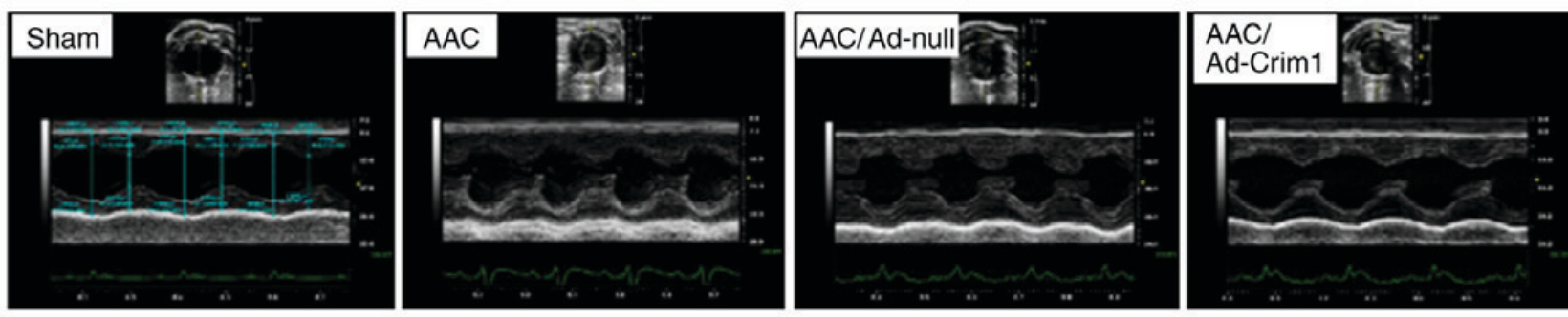

B

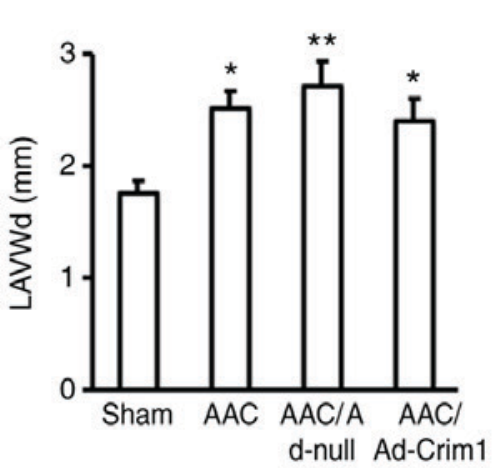

C
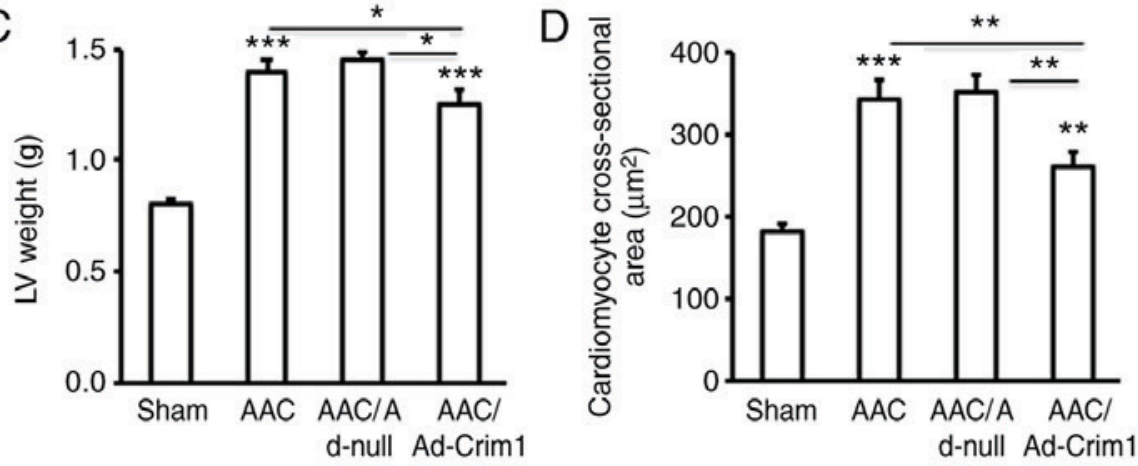
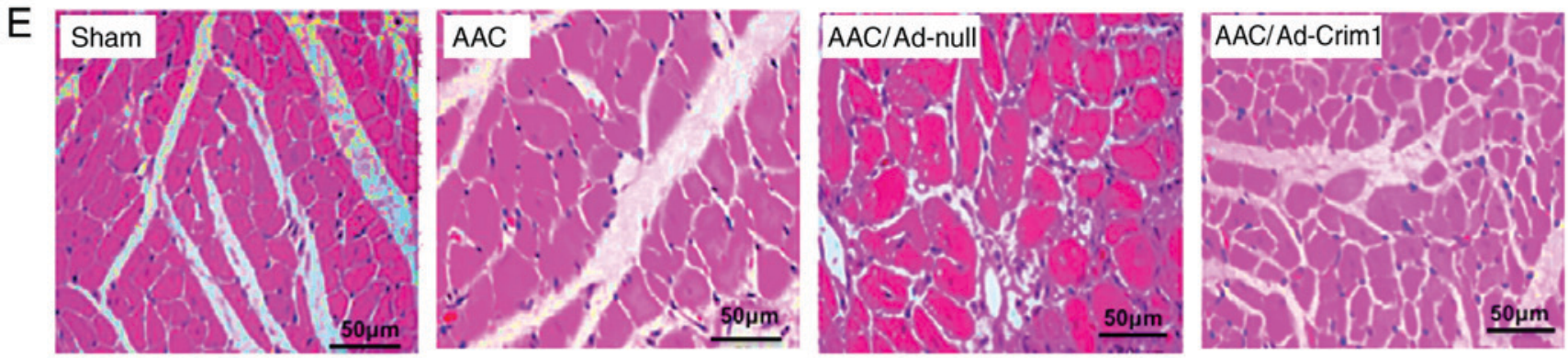

Figure 5. Effects of Ad-Crim1 on left ventricular hypertrophy in vivo. (A) AAC-treated rats were subjected to thoracic intramuscular injection of Ad-Crim1, Ad-null, or saline. Sham group rats received no treatment. Rats in the four groups were examined by echocardiography. (B) LAVWd and (C) LV weight were calculated and presented. (D) Cross-sectional areas of left ventricular myocytes were analyzed using ImageJ software. (E) Paraffin-fixed left ventricular sections were subjected to hematoxylin and eosin staining and images were captured under a confocal microscope (magnification, $\mathrm{x} 400, \mathrm{scale}$ bar $=50 \mu \mathrm{m})$. Data are from three independent experiments, presented as the mean $\pm \mathrm{SD}$, and analyzed using one-way ANOVA. ${ }^{*} \mathrm{P}<0.05,{ }^{* *} \mathrm{P}<0.01,{ }^{* * *} \mathrm{P}<0.001$. Individual treatment was compared with sham; AAC/Ad-Crim1 treatment was also compared with AAC or AAC/Ad-null treatment. Crim1, cysteine-rich transmembrane bone morphogenetic protein regulator 1; AAC, abdominal aortic coarctation; LAVWd, left ventricular end-diastolic anterior wall; LV, left ventricle.

hypertension and cardiac hypertrophy through either the AngII-dependent or AngII-independent pathway (19,32-34). The inhibition of AT1R attenuates or reverses myocardial hypertrophy $(20,35)$. In the present study, data showed that AT1R antagonist telmisartan inhibited the cardiomyocyte hypertrophy induced by stretch or AAC. Of note, stretch or AAC treatment significantly reduced the expression of Crim1 in hypertrophic ventricular myocytes. By contrast, inhibiting AT1R signaling by telmisartan significantly increased the expression of Crim1 in cardiomyocytes, suggesting a potential suppressive role of AT1R signal on the expression of Crim1 in cardiomyocytes.

In conclusion, the present study showed that the expression of Crim1 was downregulated in hypertrophic ventricular myocytes. The increased expression of Crim1 by inhibiting AT1R or the intramuscular injection of Crim1-expressing recombinant adenovirus prevented left ventricular hypertrophy. The effect of Crim1 on left ventricular hypertrophy supports the view that Crim1 can be utilized as a novel therapeutic target for the treatment of cardiac hypertrophy.

\section{Acknowledgements}

The authors would like to thank Dr Ryan Jajosky (Department of Pathology and Laboratory Medicine, Emory University School of Medicine) for editing the manuscript.

\section{Funding}

This study was supported by grants from the Guizhou Provincial Science and Technology Fund [grant no. (2019) 1209], the Chinese National Natural Science Foundation to YL (grant no. 81260040), the Chinese National Clinical Key Specialty Construction Project to YL [grant no. (2013) 544] and the Clinical Research Center Project of Department of Science \& Technology of Guizhou Province, China to YL [grant no. (2017) 5405].

\section{Availability of data and materials}

All data obtained in this study are included in this published article. 


\section{Authors' contributions}

LY and JH designed the experiments, LY, JH, GX, JY, QT and YY performed the experiments, LY, JH, JY and JD analyzed and interpreted data, LY and JD wrote the manuscript. All authors read and approved the final manuscript.

\section{Ethical approval and consent to participate}

The Animal Care Committee of Guizhou Provincial People's Hospital approved the animal experimental protocol.

\section{Patient consent for publication}

Not applicable.

\section{Competing interests}

The authors declare that they have no competing interests.

\section{References}

1. Tham YK, Bernardo BC, Ooi JY, Weeks KL and McMullen JR Pathophysiology of cardiac hypertrophy and heart failure: Signaling pathways and novel therapeutic targets. Arch Toxicol 89: 1401-1438, 2015.

2. Soliman EZ and Prineas RJ: Antihypertensive therapies and left ventricular hypertrophy. Curr Hypertens Rep 19: 79, 2017.

3. Ikeda Y, Kumagai H, Motozawa Y, Suzuki J and Komuro I: Biased agonism of the angiotensin II type I receptor. Int Heart J 56: 485-488, 2015.

4. Wang S, Gong H, Jiang G, Ye Y, Wu J, You J, Zhang G, Sun A, Komuro I, Ge J and Zou Y: Src is required for mechanical stretch-induced cardiomyocyte hypertrophy through angiotensin II type 1 receptor-dependent beta-arrestin2 pathways. PLoS One 9: e92926, 2014.

5. Kolle G, Georgas K, Holmes GP, Little MH and Yamada T: CRIM1, a novel gene encoding a cysteine-rich repeat protein, is developmentally regulated and implicated in vertebrate CNS development and organogenesis. Mech Dev 90: 181-193, 2000.

6. Glienke J, Sturz A, Menrad A and Thierauch KH: CRIM1 is involved in endothelial cell capillary formation in vitro and is expressed in blood vessels in vivo. Mec Dev 119: 165-175, 2002.

7. Wilkinson L, Kolle G, Wen D, Piper M, Scott J and Little M: CRIM1 regulates the rate of processing and delivery of bone morphogenetic proteins to the cell surface. J Biol Chem 278 : 34181-34188, 2003.

8. Nakashima Y and Takahashi S: Induction of cysteine-rich motor neuron 1 mRNA expression in vascular endothelial cells. Biochem Biophys Res Commun 451: 235-238, 2014.

9. Iyer S, Pennisi DJ and Piper M: Crim1-, a regulator of developmental organogenesis. Histol Histopathol 31: 1049-1057, 2016.

10. Iyer S, Chou FY, Wang R, Chiu HS, Raju VK, Little MH, Thomas WG, Piper M and Pennisi DJ: Crim1 has cell-autonomous and paracrine roles during embryonic heart development. Sci Rep 6: 19832, 2016.

11. Pennisi DJ, Wilkinson L, Kolle G, Sohaskey ML, Gillinder K, Piper MJ, McAvoy JW, Lovicu FJ and Little MH Crim1KST264/KST264 mice display a disruption of the Crim1 gene resulting in perinatal lethality with defects in multiple organ systems. Dev Dyn 236: 502-511, 2007.

12. Chiu HS, York JP, Wilkinson L, Zhang P, Little MH and Pennisi DJ: Production of a mouse line with a conditional Criml mutant allele. Genesis 50: 711-716, 2012.

13. Golden HB, Gollapudi D, Gerilechaogetu F, Li J, Cristales RJ, Peng $\mathrm{X}$ and Dostal DE: Isolation of cardiac myocytes and fibroblasts from neonatal rat pups. Methods Mol Biol 843: 205-214, 2012.

14. Wang GJ, Yao YS and Wang HX: Comparing effects of U50488H, prazosin and/or propranolol on cardiac hypertrophy induced by NE in rat. Zhongguo Ying Yong Sheng Li Xue Za Zhi 26: 82-85, 2010 (In Chinese).
15. Yang $\mathrm{Y}$, Zhang $\mathrm{H}$, Li X, Yang $\mathrm{T}$ and Jiang Q: Effects of PPAR $\alpha / P G C-1 \alpha$ on the energy metabolism remodeling and apoptosis in the doxorubicin induced mice cardiomyocytes in vitro. Int J Clin Exp Pathol 8: 12216-12224, 2015.

16. Livak KJ and Schmittgen TD: Analysis of relative gene expression data using real-time quantitative PCR and the 2(-Delta Delta C(T)) method. Methods 25: 402-408, 2001.

17. Deng J, Pennati A, Cohen JB, Wu Y, Ng S, Wu JH, Flowers CR and Galipeau J: GIFT4 fusokine converts leukemic B cells into immune helper cells. J Transl Med 14: 106, 2016.

18. Huang J, Wang D, Zheng J, Huang X and Jin H: Hydrogen sulfide attenuates cardiac hypertrophy and fibrosis induced by abdominal aortic coarctation in rats. Mol Med Rep 5: 923-928, 2012.

19. Sadoshima J, Xu Y, Slayter HS and Izumo S: Autocrine release of angiotensin II mediates stretch-induced hypertrophy of cardiac myocytes in vitro. Cell 75: 977-984, 1993.

20. Arumugam S, Sreedhar R, Thandavarayan RA, Karuppagounder V, Krishnamurthy P, Suzuki K, Nakamura M and Watanabe K: Angiotensin receptor blockers: Focus on cardiac and renal injury. Trends Cardiovasc Med 26: 221-228, 2016.

21. Wilkinson L, Gilbert T, Kinna G, Ruta LA, Pennisi D, Kett M and Little MH: Crim1KST264/KST264 mice implicate Crim1 in the regulation of vascular endothelial growth factor-A activity during glomerular vascular development. J Am Soc Nephrol 18: 1697-1708, 2017.

22. Fan J, Ponferrada VG, Sato T, Vemaraju S, Fruttiger M, Gerhardt H, Ferrara N and Lang RA: Crim1 maintains retinal vascular stability during development by regulating endothelial cell Vegfa autocrine signaling. Development 141: 448-459, 2014.

23. Sun B, Huo R, Sheng Y, Li Y, Xie X, Chen C, Liu HB, Li N, Li CB, Guo WT, et al: Bone morphogenetic protein-4 mediates cardiac hypertrophy, apoptosis, and fibrosis in experimentally pathological cardiac hypertrophy. Hypertension 61: 352-360, 2013.

24. Sun B, Sheng Y, Huo R, Hu CW, Lu J, Li SL, Liu X, Wang YC and Dong DL: Bone morphogenetic protein-4 contributes to the down-regulation of Kv4.3 K+ channels in pathological cardiac hypertrophy. Biochem Biophys Res Commun 436: 591-594, 2013.

25. Shahid M, Spagnolli E, Ernande L, Thoonen R, Kolodziej SA, Leyton PA, Cheng J, Tainsh RE, Mayeur C, Rhee DK, et al: BMP type I receptor ALK2 is required for angiotensin II-induced cardiac hypertrophy. Am J Physiol Heart Circ Physiol 310: H984-H994, 2016.

26. Izumiya Y, Shiojima I, Sato K, Sawyer DB, Colucci WS and Walsh K: Vascular endothelial growth factor blockade promotes the transition from compensatory cardiac hypertrophy to failure in response to pressure overload. Hypertension 47: 887-893, 2006.

27. Ferrara N: Vascular endothelial growth factor. Arterioscler Thromb Vasc Biol 29: 789-791, 2009.

28. Wilkinson L, Gilbert T, Sipos A, Toma I, Pennisi DJ, Peti-Peterdi J and Little MH: Loss of renal microvascular integrity in postnatal Crim1 hypomorphic transgenic mice. Kidney Int 76: 1161-1171,2009.

29. Zhang Y, Fan J, Ho JW, Hu T, Kneeland SC, Fan X, Xi Q, Sellarole MA, de Vries WN, Lu W, et al: Crim1 regulates integrin signaling in murine lens development. Development 143: 356-366, 2016.

30. Ross RS and Borg TK: Integrins and the myocardium. Circ Res 88: 1112-1119, 2001.

31. Israeli-Rosenberg S, Manso AM, Okada $\mathrm{H}$ and Ross RS: Integrins and integrin-associated proteins in the cardiac myocyte. Circ Res 114: 572-586, 2014.

32. Takezako T, Unal H, Karnik SS and Node K: Current topics in angiotensin II type 1 receptor research: Focus on inverse agonism, receptor dimerization and biased agonism. Pharmacol Res 123: 40-50, 2017.

33. Zou Y, Akazawa H, Qin Y, Sano M, Takano H, Minamino T, Makita N, Iwanaga K, Zhu W, Kudoh S, et al: Mechanical stress activates angiotensin II type 1 receptor without the involvement of angiotensin II. Nat Cell Biol 6: 499-506, 2004.

34. Akazawa $\mathrm{H}$ and Komuro I: Mechanisms underlying angiotensin II-independent activation of angiotensin II type 1 receptor. Nihon Rinsho 70: 1492-1498, 2012 (In Japanese).

35. Dargad RR, Parekh JD, Dargad RR and Kukrety S: Azilsartan: Novel angiotensin receptor blocker. J Assoc Physicians India 64: 96-98, 2016. 